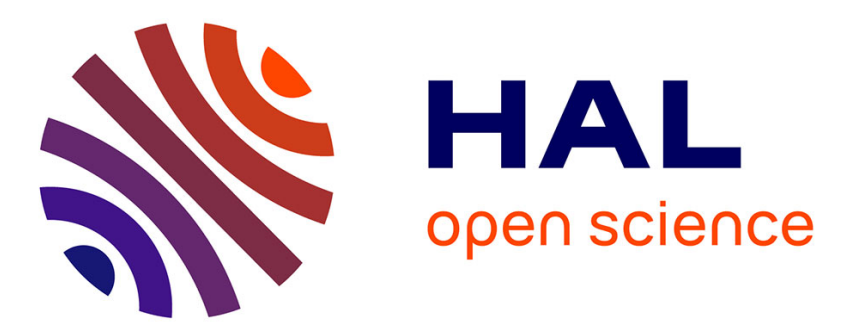

\title{
On linking microsimulation and computable general equilibrium models using exact aggregation of heterogeneous discrete-choice making agents
}

Riccardo Magnani, Jean Mercenier

\section{- To cite this version:}

Riccardo Magnani, Jean Mercenier. On linking microsimulation and computable general equilibrium models using exact aggregation of heterogeneous discrete-choice making agents. Economic Modelling, 2009, 26 (3), pp.560-570. halshs-00627736

\section{HAL Id: halshs-00627736 \\ https://shs.hal.science/halshs-00627736}

Submitted on 5 Oct 2011

HAL is a multi-disciplinary open access archive for the deposit and dissemination of scientific research documents, whether they are published or not. The documents may come from teaching and research institutions in France or abroad, or from public or private research centers.
L'archive ouverte pluridisciplinaire HAL, est destinée au dépôt et à la diffusion de documents scientifiques de niveau recherche, publiés ou non, émanant des établissements d'enseignement et de recherche français ou étrangers, des laboratoires publics ou privés. 


\title{
On Linking Microsimulation and Computable General Equilibrium Models Using Exact Aggregation of Heterogeneous Discrete-choice Making Agents
}

\author{
Riccardo Magnani* and Jean Mercenier ${ }^{\dagger} \ddagger$ \\ First version: November 2007. First revision: August 2008 \\ Second revision: November 2008
}

\begin{abstract}
Our paper contributes by bridging the gap between the (partial equilibrium) microsimulation and the computable general equilibrium (CGE) approaches, by making use of exact aggregation results from the discrete choice literature: heterogeneous individuals choosing within a set of discrete alternatives may be aggregated into a representative agent with (possibly multiple-level) constant elasticity-of-substitution/transformation preferences/technologies. These results therefore provide a natural link between the two policy evaluation approaches. We illustrate the usefulness of these results by evaluating potential effects of population ageing on the dynamics of income distribution and inequalities, using a simple overlapping generations model where individuals make leisure/work decisions, and choose a profession among a discrete set of alternatives.
\end{abstract}

JEL classification: C63; C68; C81; D31; D58; E17; J10; J22.

Keywords: Microsimulation; CGE models; Exact aggregation; Discrete choice; Nested multinomial logit, Population ageing; Income inequality.

\footnotetext{
${ }^{*}$ CEPII, 9 rue Georges Pitard, 75015 Paris. <Riccardo.Magnani@cepii.fr>

${ }^{\dagger}$ ERMES (CNRS), Université Panthéon-Assas (Paris 2), 12 place du Panthéon, 75230 Paris Cedex 05. http://mercenier.u-paris2.fr/

${ }^{\ddagger}$ We wish to thank Nathalie Picard for very helpful discussions. We express our gratitude to anonymous referees for comments that helped us improve the paper, and (without implicating) Human Resources and Social Development Canada for financial support. The usual disclaimer applies.
} 


\section{Introduction}

During the last twenty years, computable general equilibrium (CGE) models have become standard tools of quantitative policy assessment. Their appeal has built on their rigorous grounding in economic theory: agents' decision-making behaviour is derived from explicit optimisation under strictly specified technological or budget constraints, given market signals that ensure global consistency. These theoretical foundations have made CGE models appear particularly useful for ex-ante evaluations of policy reforms. However, the whole apparatus relies on the concept of "representative agent" despite unclear aggregation procedures to link these aggregate optimising decision-makers to the numerous individual agents whose behaviour they are meant to capture.

During the same period, another class of models has become increasingly popular: behavioural microsimulation models. Their appeal stems from the fact that they avoid any reliance on typical agents by fully taking into account the heterogeneity of individual choices as they are revealed in micro-data sets. ${ }^{1}$ Indeed, working with myriads of actual economic agents rather than with a few hypothetical ones makes it possible to precisely identify the winners and the losers of a reform - obviously a major concern to policymakers - yet, making it possible by simple addition to accurately measure this impact on aggregate variables. The increasing availability of large and detailed data sets on individuals makes this quite appealing. The drawback of the approach is that it is partial equilibrium in essence: for instance, individual's labour supply adjustment to some new tax incentive scheme can be quite accurately captured for given wages and other policy parameters, but market equilibrium and government budget constraints can be expected to have a feedback influence on the same individual's choices that is typically neglected. One could of course imagine iterating between the microsimulation and the CGE models, and indeed, a few efforts have successfully been done in this direction: see for instance Savard (2003) and the elaboration of Arntz et al. (2008) on Arntz et al. (2006). Though this iterative strategy might be satisfactory for some problems - in particular when dynamics are thought unimportant - it becomes tedious for more sophisticated apparatus such as overlapping generations (OLG) models: see however Rausch and Rutherford (2007) for

\footnotetext{
${ }^{1}$ See Bourguignon and Spadaro (2006) for an excellent survey and an extensive list of references.
} 
progress in that direction.

In this paper, we make use of simple yet powerful exact aggregation results due to Anderson, de Palma and Thisse (1992) (here after: AdPT) who show that, under reasonably mild conditions, heterogeneous individuals that have to choose (possibly continuous amounts) within a set of discrete alternatives may be aggregated into a representative agent with constant elasticity-of-substitution (CES) preferences. ${ }^{2}$ We illustrate how these results can be useful to CGE modellers by making available to them a growing body of empirical estimates from microeconometrics that can be used to parameterise CES/CET (constant elasticity-of-transformation) preferences/technologies in the representative agent framework. Furthermore, we argue that these results provide a natural and appealing link between the standard CGE apparatus and the microsimulations approach, and suggest that they constitute a useful alternative approach to the iterative strategy between microsimulation and CGE models. There is no free lunch, unfortunately: some details captured by the microsimulation approach could be lost, a cost that one should balance against the benefits of accounting for the general equilibrium feedbacks.

We show how to make use of these results in order to link the micro and the macro simulation approaches, and illustrate the usefulness of the methodology in the context of population ageing using a calibrated overlapping generations (OLG) model. For this, we first generate in vitro a micro-data set where individuals, classified in different cells according to their socio-economic characteristics, face random utility maximisation problems over sets of discrete alternatives. We focus, for illustrative purposes, on labour market participation, and particularise the discrete choices as "to work or not to work, and if work is chosen, in which profession?" in a nested multinomial logit framework. ${ }^{3}$ We then show that the aggregation of individual choices yields a labour-supply scheme that coincides with the one derived from a macro-agent's time-allocation problem subject to smooth nested CES preferences as typically used in CGE models. The representative agent is part of a dynamic GE model which we simulate to evaluate the effects of a demographic

\footnotetext{
${ }^{2}$ Discrete choice models can be extended to so-called continuous/discrete models that allow individuals to demand continuous quantities (not restricted to 0 or 1 ) of their preferred discrete option. See, e.g., Train (1986, Chap. 5).

${ }^{3}$ At the risk of being overemphatic, it seems useful to insist that the aggregation methodology is quite general and can be applied to a broad set of choices other than labour supply decisions.
} 
shock on the time path of wages and interest rates. These equilibrium prices are then plugged into the microsimulation model in order to determine the response of each individual micro-agent to the changes in his/her economic environment. From this individual choice response, we can compute the income distributions consistent with general equilibrium wages, and therefore apprehend the dynamics of income inequalities induced by population ageing.

The paper is organised as follows: in Section 2, we provide a refresher on probabilistic discrete choice models. Focusing on a typical labour force participation decision problem, we show in Section 3 how to link the myriads of heterogeneous micro-agents of the microsimulation approach to a macro-agent. This macro-agent is embedded in the dynamic GE model sketched in Section 4. We then submit in Section 5 the OLG economy to an ageing shock, and plug the equilibrium prices in the microsimulation model to generate the time-path of income inequality indicators. The paper closes with a brief conclusion.

\section{Discrete-choice models: a refresher}

Assume a population of individuals $h=1, \ldots, N$ has to choose among a set $i, j=0, \ldots, I$ of discrete alternatives with associated utility levels:

$$
\widetilde{u}_{i}^{h}=u_{i}+\epsilon_{i}^{h} \quad i=0, \ldots, I
$$

where $u_{i}$ is a deterministic component (for now, assumed common to all individuals) and $\epsilon_{i}^{h}$ is a random term. Each $h$ is therefore characterised by a draw $\epsilon=\left(\epsilon_{0}^{h}, \ldots, \epsilon_{I}^{h}\right)$ in a probability distribution with cumulative distribution function $F(\epsilon)$. Assume that individuals in this population are not only statistically identical but also statistically independent. Then, the distribution of choices is multinomial with mean $\bar{X}_{i}=N P_{i}$, $i=0, \ldots, I$, where $P_{i}$ denotes the probability that alternative $i$ be chosen by $h . \bar{X}_{i}$ is the mathematical expectation of demand for alternative $i$; for $N$ large enough, $\bar{X}_{i}$ is a close approximation of aggregate demand for $i$ in this population. In other words, aggregate demands for each alternative may be readily determined from the choice probabilities from the individual discrete decision problem. 
The probability that $h$ will choose alternative $i$ is:

$$
\begin{aligned}
P_{i} & =\operatorname{prob}\left[\widetilde{u}_{i}^{h} \geq \widetilde{u}_{j}^{h}, \forall j=0, \ldots, I\right] \\
& =\operatorname{prob}\left[u_{i}+\epsilon_{i}^{h} \geq u_{j}+\epsilon_{j}^{h}, \forall j=0, \ldots, I\right] \\
& =\operatorname{prob}\left[\epsilon_{j}^{h}-\epsilon_{i}^{h} \leq u_{i}-u_{j}, \forall j=0, \ldots, I\right]
\end{aligned}
$$

The determination of the choice probabilities using $F(\epsilon)$ is in principle always possible but in general extremely difficult, in particular if $\epsilon$ is assumed normally distributed as would seem natural. Fortunately, a theorem due to McFadden ${ }^{4}$ identifies a class of cumulative distribution functions $F(\epsilon)$ - of which the double exponential is a special case that yields the multinomial logit - for which these probabilities may be easily determined indirectly. Consider the multivariate generalised extreme value (GEV) cumulative distribution function

$$
F\left(\epsilon_{0}, \ldots, \epsilon_{I}\right)=\exp \left[-H\left(e^{-\epsilon_{0}}, \ldots, e^{-\epsilon_{I}}\right)\right]
$$

with $H$ a nonnegative function defined over $R_{+}^{N}$ satisfying the following properties:

$H$ is homogeneous of degree $1 / \mu ; \quad(i i) \lim _{x_{i} \rightarrow \infty} H\left(x_{0}, \ldots, x_{I}\right)=\infty \forall i=0, \ldots, I ; \quad$ (iii) the mixed partial derivatives of $H$ with respect to $k$ different variables exist and are continuous, non-negative if $k$ is odd, non-positive if $k$ is even, $k=0, \ldots, I$. (These technical conditions are needed to ensure that $F(\epsilon)$ is indeed a cumulative distribution function.) Then, McFadden's GEV theorem states that the choice probabilities $P_{i}$ may be determined as:

$$
P_{i}=\mu \frac{\partial \ln H\left(e^{u_{0}}, \ldots, e^{u_{I}}\right)}{\partial u_{i}}
$$

Many particularisations of $H$ consistent with utility maximisation are possible, and to each corresponds a different distribution for $\epsilon$. One important specification for $H$ is:

$$
H\left(e^{-\epsilon_{0}}, \ldots, e^{-\epsilon_{I}}\right)=\sum_{i=0}^{I} e^{-\frac{\epsilon_{i}}{\mu}}
$$

It is easily checked that this function satisfies the properties of the theorem; the associated GEV cumulative distribution function writes as:

$$
F\left(\epsilon_{0}, \ldots, \epsilon_{I}\right)=\exp \left[-\sum_{i=0}^{I} e^{-\frac{\epsilon_{i}}{\mu}}\right]=\prod_{i=0}^{I} \exp \left[-e^{-\frac{\epsilon_{i}}{\mu}}\right]
$$

\footnotetext{
${ }^{4}$ See McFadden 1978, p.80; 1981, p.227.
} 
and is therefore the product of $I+1$ i.i.d. double-exponential (or extreme value), with dispersion parameter $\mu$, which apply to the stochastic utilities $\widetilde{u}_{i}$ in (1). It follows from (4) that

$$
P_{i}=\mu \frac{\partial \ln \sum_{j=0}^{I} e^{\frac{u_{j}}{\mu}}}{\partial u_{i}}=\frac{e^{\frac{u_{i}}{\mu}}}{\sum_{j=0}^{I} e^{\frac{u_{j}}{\mu}}}
$$

which are the familiar choice probabilities derived from a multinomial-logit population. The simplicity of this formula obviously makes the MNL quite appealing. It turns out that, in addition, it provides a good approximation to the normal distribution. ${ }^{5}$

Preferences (1) with i.i.d. double-exponential random terms have the special property that the ratio of the probabilities between two alternatives are the same no matter what other alternatives are available or what the attributes of the other alternatives are. ${ }^{6}$ This property, known as the independence from irrelevant alternatives may be acceptable in some problems, but is clearly over-restrictive when some alternatives are closer to each others within a group than to others outside that group. ${ }^{7}$ It can, fortunately, easily be bypassed by nesting multinomial logit systems, as we now show.

Assume that the set of alternatives $i, j=0, \ldots, I$ can be partitioned into $M+1$ nonoverlapping subsets $\left\{A_{m} ; m=0, \ldots, M\right\}$ of close alternatives called nests. Preferences (1) still apply with random terms distributed as extreme value, but they are no longer independent. Rather, they are assumed positively correlated across alternatives in each nest: if $h$ has a high value for $\epsilon_{j}^{h} j \in A_{m}$, then $h$ is also likely to value highly other options in

\footnotetext{
${ }^{5}$ Ben Akiva and Lerman (1985, p.128) write: "there is still no evidence to suggest in which situations the greater generality of the multinomial probit is worth the additional computational problems resulting from its use." We are not aware that such evidence has been reported in the literature since then.

${ }^{6}$ Observe that any change in the deterministic utility level associated with alternative $j$ will affect symmetrically the choice probabilities of all other alternatives: from (7),

$$
\frac{\partial P_{i}}{\partial u_{j}}=-\frac{P_{i} P_{j}}{\mu} \quad i, j=0, \ldots, I \quad i \neq j
$$
}

so that the cross-elasticities

$$
\operatorname{Elas}\left(P_{i}, u_{j}\right)=-\frac{P_{j} u_{j}}{\mu} \quad i, j=0, \ldots, I \quad i \neq j
$$

are independent of $i$.

${ }^{7}$ For more on the implications of the property of independence from irrelevant alternatives, see e.g. Train (2003, p.49). 
nest $A_{m}$. We particularise the $H\left(e^{-\epsilon_{0}}, \ldots, e^{-\epsilon_{I}}\right)$ function as follows:

$$
H\left(e^{-\epsilon_{0}}, \ldots, e^{-\epsilon_{I}}\right)=\sum_{m=0}^{M}\left[\sum_{j \in A_{m}} e^{-\frac{\epsilon_{j}}{\mu_{2}}}\right]^{\frac{\mu_{2}}{\mu_{1}}}
$$

Observe that if $\mu_{1}=\mu_{2},(8)$ is identical to (5). This $H$ function is homogeneous of degree $1 / \mu_{1}$; McFadden (1981) has shown that if $\mu_{1} \geq \mu_{2}$, it satisfies all the properties required to apply the extreme value theorem: the associated GEV cumulative distribution function is

$$
F\left(\epsilon_{0}, \ldots, \epsilon_{I}\right)=\exp \left\{-\sum_{m=0}^{M}\left[\sum_{j \in A_{m}} e^{-\frac{\epsilon_{j}}{\mu_{2}}}\right]^{\frac{\mu_{2}}{\mu_{1}}}\right\}
$$

where $\frac{\mu_{2}}{\mu_{1}}$ is a rough measure of the correlation between random terms within a nest (see Ben-Akiva and Lerman, 1985, p.289). Using (4), we compute the probability that, among all alternatives, option $i$ of nest $A_{m}$ be chosen as:

$$
\begin{aligned}
P_{i} & =\mu_{1} \frac{\partial \ln \sum_{m=0}^{M}\left[\sum_{j \in A_{m}} e^{\frac{u_{j}}{\mu_{2}}}\right]^{\frac{\mu_{2}}{\mu_{1}}}}{\partial u_{i}} \\
= & \frac{\left[\sum_{j \in A_{m}} e^{\frac{u_{j}}{\mu_{2}}}\right]^{\frac{\mu_{2}}{\mu_{1}}}}{\sum_{m=0}^{M}\left[\sum_{j \in A_{m}} e^{\frac{u_{j}}{\mu_{2}}}\right]^{\frac{\mu_{2}}{\mu_{1}}}} \frac{e^{\frac{u_{i}}{\mu_{2}}}}{\sum_{j \in A_{m}} e^{\frac{u_{j}}{\mu_{2}}}} \quad i \in A_{m}
\end{aligned}
$$

This expression has a structure that makes it quite intuitive. The second term is the probability that $h$ will choose alternative $i \in A_{m}$ conditional on having already chosen nest $A_{m}$. The first term represents the probability of choosing any option from $A_{m}$. It can easily be checked that the property of independence from irrelevant alternatives holds within each subset of alternatives but not across subsets (see e.g. Train, 2003, p.84).

Expression (10) can be given an alternative welfare interpretation that will prove useful. To see this, define function $H_{A_{m}}$ on subset $A_{m}$ as:

$$
H_{A_{m}}=H_{A_{m}}\left(e^{-\epsilon_{j}}, j \in A_{m}\right)=\sum_{j \in A_{m}} e^{-\frac{\epsilon_{j}}{\mu_{2}}}
$$

so that, within each nest, preferences are given by (1) with double exponential random terms. It can be shown (see e.g. AdPT, p.60) that the expected value of the maximum of 
utilities from the alternatives in nest $A_{m}$ is:

$$
G_{A_{m}}=G_{A_{m}}\left(u_{j}, j \in A_{m}\right)=\mu_{2} \ln \sum_{j \in A_{m}} e^{\frac{u_{j}}{\mu_{2}}}
$$

$G_{A_{m}}$ can be interpreted as a measure of the attractiveness, or the utility valuation, of the subset of options $A_{m}$. Observe that, dividing $G_{A_{m}}$ by $\mu_{1}$ and applying an exponential transform, yields:

$$
e^{\frac{G_{A_{m}}}{\mu_{1}}}=\left[\sum_{j \in A_{m}} e^{\frac{u_{j}}{\mu_{2}}}\right]^{\frac{\mu_{2}}{\mu_{1}}}
$$

Next, define ${ }^{8}$

$$
\varepsilon_{A_{m}}^{h}=\max _{j \in A_{m}}\left(u_{j}+\epsilon_{j}^{h}\right)-G_{A_{m}}
$$

and the random preferences for choosing between nests as:

$$
\widetilde{u}_{A_{m}}^{h}=G_{A_{m}}+\varepsilon_{A_{m}}^{h} \quad m=0, \ldots, M
$$

where $G_{A_{m}}$ is given by (12). Let $H\left(e^{-\varepsilon_{A_{0}}}, \ldots, e^{-\varepsilon_{A_{M}}}\right)$ be of the now familiar form

$$
H\left(e^{-\varepsilon_{A_{0}}}, \ldots, e^{-\varepsilon_{A_{M}}}\right)=\sum_{m=0}^{M} e^{-\frac{\varepsilon_{A_{m}}}{\mu_{1}}}
$$

We know that such a function satisfies the properties of the GEV theorem so that the choice probability for option $m$-that is, for nest $A_{m}$ - is immediately obtained using (4) as:

$$
\begin{aligned}
P_{A_{m}} & =\mu_{1} \frac{\partial \ln H\left(e^{G_{A_{0}}}, \ldots, e^{G_{A_{M}}}\right)}{\partial G_{A_{m}}} \\
& =\frac{e^{\frac{G_{A_{m}}}{\mu_{1}}}}{\sum_{m \prime=0}^{M} e^{\frac{G_{A_{m \prime} \prime}}{\mu_{1}}}} \\
& =\frac{\left[\sum_{j \in A_{m}} e^{\frac{u_{j}}{\mu_{2}}}\right]^{\frac{\mu_{2}}{\mu_{1}}}}{\sum_{m /=0}^{M}\left[\sum_{j \in A_{m}} e^{\frac{u_{j}}{\mu_{2}}}\right]^{\frac{\mu_{2}}{\mu_{1}}}}
\end{aligned}
$$

where use has been made of (13). A comparison of this result with (10), makes it clear that the probability of choosing an option $i$ that belongs to a specific nest $A_{m}$ can be

\footnotetext{
${ }^{8}$ See Ben-Akiva and Lerman (1985, p.288).
} 
given a very intuitive structure:

$$
P_{i}=\frac{e^{\frac{G_{A_{m}}}{\mu_{1}}}}{\sum_{m /=0}^{M} e^{\frac{G_{A_{m} /}}{\mu_{1}}}} \cdot \frac{e^{\frac{u_{i}}{\mu_{2}}}}{\sum_{j \in A_{m}} e^{\frac{u_{j}}{\mu_{2}}}} \quad i \in A_{m}
$$

where the first term is a logit choice probability between the $M+1$ subsets of alternatives, each nest being utility valued by the expected maximum utility from those alternatives that belong to it.

The discrete choice preferences that give rise to these decision probabilities may be conveniently given a nested form:

$$
\begin{cases}\widetilde{u}_{A_{m}}^{h}=G_{A_{m}}+\varepsilon_{A_{m}}^{h} & m=0, \ldots, M \\ \widetilde{u}_{i}^{h}=u_{i}+\epsilon_{i}^{h} & i \in A_{m}\end{cases}
$$

where $G_{A_{m}}$ is given by (12) and $\varepsilon_{A_{m}}^{h}$ by (14). Nested discrete choice decision problems can therefore quite simply be solved sequentially, one level after the other, up the decision tree: it is immediate to generalise this to any number $q$ of nesting levels, provided that $\mu_{1} \geq \mu_{2} \geq \ldots \geq \mu_{q}$ where $q$ is the lowest level in the decision tree, i.e. where individual heterogeneity is lowest. ${ }^{9}$

\section{Modelling leisure/work decisions and the choice of a pro- fession}

Many applications of behavioural microsimulation models are related to labour supply decisions and, for this reason, we illustrate in this section the use of the discrete choice methodology to model labour market participation. More specifically, we consider the following individual nested decisions problem: should I work or not, and if I do, which profession should I choose? Each individual discrete decision will be conditional on some prices (in the current example, wages) and possibly on some policy parameters (such as tax rates) that are typically exogenous to the myriads of decisions-makers who constitute the microsimulation model. To endogenise those prices and possibly budget-induced tax-rate adjustments requires a general equilibrium set-up with fewer macro-agents representative

\footnotetext{
${ }^{9}$ See Ben-Akiva and Lerman, 1985, p.293. Note that our parameter $\mu$ has the inverse definition of theirs.
} 
of the underlying micro-population behaviour: we next provide such a representative agent formulation that exactly replicates the sum of individual labour supply decisions.

\subsection{The individual discrete choice formulation}

The population of the individuals in the micro-data set is partitioned into $z=1, \ldots, Z$ cells according to as many characteristics as available, such as sex, age-class etc. In what follows, we model the decision problems of individuals belonging to one such cell, and neglect the subscript $z$ to ease notation. In the applied general equilibrium model there will be one macro-agent for each cell.

Consider $h$ belonging to the cell, therefore belonging to a sub-population with the same socio-economic characteristics. This individual has to decide whether to work or not, and if he/she chooses to work, in which profession. We model this as a two-level discrete choice problem and take advantage of the nested structure to solve the problem sequentially starting from the lowest level of the decision tree: the choice of profession. In terms of our previous notations, the set of discrete alternatives is $I=\{$ leisure, work in profession 1, work in profession $2, \ldots$, work in profession $I\}$. The postulated two-level nested structure implies partitioning $I$ into two subsets: $A_{0}=\{$ leisure $\}$ and $A_{1}=\{$ work in profession $1, \ldots$, work in profession $I\}$.

\subsubsection{Choosing between professions}

We write the utility of choosing profession $i \in A_{1}$ as a log-linear function:

$$
\widetilde{u}_{i}^{h}=\ln \theta_{i}+\ln \bar{w}_{i}+\epsilon_{i}^{h} \quad i \in A_{1}
$$

The first term, $\theta_{i}$, captures both the disutility of working (common to all options in $A_{1}$ ) and the (dis)utility specific to profession $i$; $\bar{w}_{i}$ is the within-cell average market wage (typically adjusted for the cell's specific efficiency level) expressed in terms of the consumption good. Note that these two terms are common to all $h$ within the considered population cell. We therefore assume here that, upon making their optimal decisions, individuals ignore possible within-cell idiosyncratic productivity differences, that will ex-post be respons- 
ible for the observed within-cell distribution of wages in the data. ${ }^{10}$ Intra-cell individual heterogeneity in preferences is captured by the (correlated) double exponential stochastic term $\epsilon_{i}^{h} i \in A_{1}$ with dispersion parameter $\mu_{2}$. From the previous section, we know that $P_{i}^{A_{1}}$, the probability $h$ will choose profession $i$ within subset $A_{1}$ is:

$$
\begin{aligned}
P_{i}^{A_{1}} & =\frac{\exp \left(\frac{\ln \theta_{i}+\ln \bar{w}_{i}}{\mu_{2}}\right)}{\sum_{j \in A_{1}} \exp \left(\frac{\ln \theta_{j}+\ln \bar{w}_{j}}{\mu_{2}}\right)} \\
& =\frac{\theta_{i}^{\frac{1}{\mu_{2}}} \cdot \bar{w}_{i}^{\frac{1}{\mu_{2}}}}{\sum_{j \in A_{1}} \theta_{j}^{\frac{1}{\mu_{2}}} \cdot \bar{w}_{j}^{\frac{1}{\mu_{2}}}} \quad i \in A_{1}
\end{aligned}
$$

\subsubsection{Choosing whether to work or not}

At the upper level of the decision tree, $h$ has to choose between $A_{0}$ and $A_{1}$ : to enjoy leisure or to work. To model this, let the utility enjoyed from not working be:

$$
\widetilde{u}_{A_{0}}^{h}=\ln \theta_{A_{0}}+\varepsilon_{A_{0}}^{h}
$$

where $\theta_{A_{0}}$ is a constant, and $\varepsilon_{A_{0}}^{h}$ is a random term which captures individual heterogeneity in the disutility of working. The utility valuation of the alternative $A_{1}$ that is consistent with the second stage decision problem is, from (18):

$$
\widetilde{u}_{A_{1}}^{h}=G_{A_{1}}+\varepsilon_{A_{1}}^{h}
$$

with $\varepsilon_{A_{1}}^{h}$ related to $\epsilon_{i}^{h} \quad i \in A_{1}$ using (14) and $G_{A_{1}}$ the expected maximum utility obtained from choosing to work:

$$
\begin{aligned}
G_{A_{1}} & =\mu_{2} \ln \sum_{j \in A_{1}} \exp \left(\frac{\ln \theta_{j}+\ln \bar{w}_{j}}{\mu_{2}}\right) \\
& =\mu_{2} \ln \sum_{j \in A_{1}} \theta_{j}^{\frac{1}{\mu_{2}}} \cdot \bar{w}_{j}^{\frac{1}{\mu_{2}}}
\end{aligned}
$$

\footnotetext{
${ }^{10}$ The additional information contained in the within-cell distribution of individual wages $w_{i}^{h}$ will of course be used in the econometric estimation of the parameters of the discrete-choice preferences, and in the microsimulations. We shall later evaluate how distortive is the substitution of $\bar{w}_{i}$ for $w_{i}^{h}$ here.
} 
$\varepsilon_{A_{0}}^{h}, \varepsilon_{A_{1}}^{h}$ are i.i.d. double exponential random terms with dispersion parameter $\mu_{1}$. The probability that $h$ will choose to work is:

$$
\begin{aligned}
P_{A_{1}} & =\frac{\exp \left(\frac{G_{A_{1}}}{\mu_{1}}\right)}{\theta_{A_{0}}^{\frac{1}{\mu_{1}}}+\exp \left(\frac{G_{A_{1}}}{\mu_{1}}\right)} \\
& =\frac{\left[\sum_{j \in A_{1}} \theta_{j}^{\frac{1}{\mu_{2}}} \cdot \bar{w}_{j}^{\frac{1}{\mu_{2}}}\right]^{\frac{\mu_{2}}{\mu_{1}}}}{\theta_{A_{0}}^{\frac{1}{\mu_{1}}}+\left[\sum_{j \in A_{1}} \theta_{j}^{\frac{1}{\mu_{2}}} \cdot \bar{w}_{j}^{\frac{1}{\mu_{2}}}\right]^{\frac{\mu_{2}}{\mu_{1}}}}
\end{aligned}
$$

\subsubsection{Total labour supply by profession from summing the individual de- cisions}

Let there be a large enough set $N$ of statistically identical and independent individuals in the sub-population cell with same socio-economic characteristics. ${ }^{11}$ Each individual owns the same amount of time, that we normalise to unity. The within-cell aggregate labour supply by profession resulting from individual discrete choices is then closely approximated by the mathematical expectation:

$$
\begin{aligned}
& L_{i}^{\text {sup }}=P_{A_{1}} \cdot P_{i}^{A_{1}} \cdot N \\
& =\frac{\exp \left(\frac{G_{A_{1}}}{\mu_{1}}\right)}{\theta_{A_{0}}^{\frac{1}{\mu_{1}}}+\exp \left(\frac{G_{A_{1}}}{\mu_{1}}\right)} \cdot \frac{\exp \left(\frac{\ln \theta_{i}+\ln \bar{w}_{i}}{\mu_{2}}\right)}{\sum_{j \in A_{1}} \exp \left(\frac{\ln \theta_{j}+\ln \bar{w}_{j}}{\mu_{2}}\right)} \cdot N \\
& =\frac{\left[\sum_{j \in A_{1}} \theta_{j}^{\frac{1}{\mu_{2}}} \cdot \bar{w}_{j}^{\frac{1}{\mu_{2}}}\right]^{\frac{\mu_{2}}{\mu_{1}}}}{\theta_{A_{0}}^{\frac{1}{\mu_{1}}}+\left[\sum_{j \in A_{1}} \theta_{j}^{\frac{1}{\mu_{2}}} \cdot \bar{w}_{j}^{\frac{1}{\mu_{2}}}\right]^{\frac{\mu_{2}}{\mu_{1}}}} \cdot \frac{\theta_{i}^{\frac{1}{\mu_{2}}} \cdot \bar{w}_{i}^{\frac{1}{\mu_{2}}}}{\sum_{j \in A_{1}} \theta_{j}^{\frac{1}{\mu_{2}}} \cdot \bar{w}_{j}^{\frac{1}{\mu_{2}}}} \cdot N \quad i \in A_{1}
\end{aligned}
$$

This is the aggregate labour force supplied in each profession, reported for given wages $\bar{w}_{i}$, by the microsimulation model.

\subsection{The aggregate representative agent formulation}

We now show that the same aggregate labour supply function (25) can be derived from the optimisation problem of a single macro-agent with nested CET (constant elasticity of

\footnotetext{
${ }^{11} N$ should be large enough -say, more than a 100 - which could limit the number of demographic characteristics that can be singled out. The size of available micro data-sets is rapidly increasing, however, so that this should not prove too much of an issue.
} 
transformation) constraints as is customarily used in CGE models. We here again neglect the cell index $z$ to ease notation, and write $N$ the total time to be split between leisure and professional activities by the macro-agent of the cell. Because nested CET functions are additively separable, we know we can solve the optimisation problem sequentially, in two steps.

We first determine the sharing of $N$ between leisure time and work time. Let $S_{\mathcal{L}}$ and $S_{L}$ denote some measure of time devoted respectively to leisure $(\mathcal{L})$ and working $(L)$. Let $\lambda_{\mathcal{L}}$ and $\lambda_{L}$ be the agent's valuation respectively of leisure and work; they are related to market wages in a way that will be established later, but are assumed given at this stage of the optimisation.

The macro-agent's problem is to choose $S_{\mathcal{L}}$ and $S_{L}$ so as to maximise the total valuation of time $\left(\lambda_{\mathcal{L}} S_{\mathcal{L}}+\lambda_{L} S_{L}\right)$ subject to a transformation constraint:

$$
\left(\alpha_{\mathcal{L}}\left[S_{\mathcal{L}}\right]^{\frac{\tau+1}{\tau}}+\alpha_{L}\left[S_{L}\right]^{\frac{\tau+1}{\tau}}\right)^{\frac{\tau}{\tau+1}}=1 \quad \tau>0
$$

The concavity of the transformation constraint is governed by the value of the transformation elasticity $\tau$; it can be interpreted as capturing the fact that moving in and out of the job market is not costless for the agent in terms of utility: the higher the value of $\tau$, the more linear is the transformation constraint and the more responsive will be the agent's optimal time allocation $S_{\mathcal{L}} / S_{L}$ to changes in $\lambda_{\mathcal{L}} / \lambda_{L}$. From the FOC, it immediately follows that the optimal time allocation satisfies:

$$
\frac{S_{\mathcal{L}}}{S_{L}}=\left[\frac{\alpha_{\mathcal{L}}}{\alpha_{L}}\right]^{-\tau} \cdot\left[\frac{\lambda_{\mathcal{L}}}{\lambda_{L}}\right]^{\tau}
$$

Defining $\lambda=\frac{\lambda_{L}}{\lambda_{\mathcal{L}}}$ the relative valuation of work with respect to leisure, and making use of (27) jointly with the resource constraint $\mathcal{L}+L=N$ yields the agent's optimal time supply on the labour market:

$$
L=\frac{\alpha_{L}^{-\tau} \lambda^{\tau}}{\alpha_{\mathcal{L}}^{-\tau}+\alpha_{L}^{-\tau} \lambda^{\tau}} \cdot N
$$

A rise in $\lambda$ enhances labour force participation.

The second step of the macro-agent's decision problem consists in allocating this work time between professions taking into account relative market wages. The fact that wages differ between professions clearly reflect differentiation by the agent. Formally, we again 
model this process as: choose allocation shares $s_{i}(i=1, \ldots, I)$ so as to maximise earned income $\sum_{i=1}^{I} w_{i} s_{i}$ subject to the following constraint with constant transformation elasticity $\sigma:$

$$
\left(\sum_{i=1}^{I} \alpha_{i} \cdot\left[s_{i}\right]^{\frac{\sigma+1}{\sigma}}\right)^{\frac{\sigma}{\sigma+1}}=1 \quad \sigma>0
$$

Again, the concavity of the transformation constraint can be interpreted as capturing the difficulty (disutility) for the macro-agent to move in and out of a profession. Solving this problem yields the optimal ratios:

$$
\frac{s_{i}}{s_{j}}=\left[\frac{\alpha_{i}}{\alpha_{j}}\right]^{-\sigma} \cdot\left[\frac{w_{i}}{w_{j}}\right]^{\sigma} \quad i, j=1, \ldots, I \quad i \neq j
$$

which, jointly with the resource constraint $\sum_{i=1}^{I} L_{i}^{\text {sup }}=L$ determines the optimal amount of time devoted to working in each profession:

$$
L_{i}^{\mathrm{sup}}=\frac{\alpha_{i}^{-\sigma} \cdot w_{i}^{\sigma}}{\sum_{j=1}^{I} \alpha_{j}^{-\sigma} \cdot w_{j}^{\sigma}} \cdot L \quad i=1, \ldots, I
$$

Making use of (28), we can substitute out $L$ to get the macro-agent's supply of labour on the market for profession $i$ :

$$
L_{i}^{\text {sup }}=\frac{\alpha_{L}^{-\tau} \cdot \lambda^{\tau}}{\alpha_{\mathcal{L}}^{-\tau}+\alpha_{L}^{-\tau} \cdot \lambda^{\tau}} \cdot \frac{\alpha_{i}^{-\sigma} \cdot w_{i}^{\sigma}}{\sum_{j=1}^{I} \alpha_{j}^{-\sigma} \cdot w_{j}^{\sigma}} \cdot N \quad i=1, \ldots, I
$$

The price aggregator $\lambda$ that expresses the agent's relative valuation of work reflects both his/her differentiation between professions and the market wages earned in those professions:

$$
\lambda=\alpha_{L}\left[\sum_{j=1}^{I} \alpha_{j}^{-\sigma} \cdot w_{j}^{\sigma}\right]^{\frac{1}{\sigma}}
$$

Substituting $\lambda$ out of (31), we obtain:

$$
L_{i}^{\sup }=\frac{\left[\sum_{j=1}^{I} \alpha_{j}^{-\sigma} \cdot w_{j}^{\sigma}\right]^{\frac{\tau}{\sigma}}}{\alpha_{\mathcal{L}}^{-\tau}+\left[\sum_{j=1}^{I} \alpha_{j}^{-\sigma} \cdot w_{j}^{\sigma}\right]^{\frac{\tau}{\sigma}}} \cdot \frac{\alpha_{i}^{-\sigma} \cdot w_{i}^{\sigma}}{\sum_{j=1}^{I} \alpha_{j}^{-\sigma} \cdot w_{j}^{\sigma}} \cdot N \quad i=1, \ldots, I
$$

Comparing this expression with (25), it is readily seen that, though the interpretation of the parameters differs considerably, the two expressions are identical provided that we set:

$$
\left\{\begin{array}{l}
\sigma=1 / \mu_{2} \\
\tau=1 / \mu_{1} \\
\alpha_{i}=1 / \theta_{i} \\
\alpha_{\mathcal{L}}=1 / \theta_{A_{0}}
\end{array} \quad i=1, \ldots, I\right.
$$


and $\bar{w}_{i}=w_{i}$.

To sum-up, we have shown that when micro-agent decision problems can be formalised as random choice multilevel logits, an aggregate representative agent can be formulated with nested CES/CET preferences/technologies that yield the same optimal decision system. Obviously, from a strict numerical perspective, this aggregate agent is by no means necessary since introducing system (25) rather than (32) into the GE model yields the same equilibrium solution. Working with a well behaved aggregate agent is however likely to be conceptually much easier and convenient to many modellers, when understanding GE policy results, than working with random utility discrete choice models of myriads of micro-agents. ${ }^{12}$

Furthermore, the reader is surely aware that there exists today an immense applied microeconometric literature based on discrete choice models that provides us with treasures of statistical information, in all fields of economics. A large fraction of that work builds on (some form of) the nested logit model. The discussion of this section suggests that CGE modellers could make use of this growing body of econometric behavioural information at very low cost, since this need not be done at the expense of their standard tool-kit: the multilevel CES/CET transform.

\subsection{The OLG set-up}

The socio-economic characteristics we consider here are age-cohorts (indexed $g$ ) and sex (indexed $s$ ). We know, from (32) that to each population cell corresponds a macro-agent with specific labour supply system indexed $i, g, s$ where as before, $i=1, \ldots, I$ is profession. ${ }^{13}$ Because both wages and the size of the cell's population change with time, we add a time

\footnotetext{
${ }^{12} \mathrm{~A}$ word of caution concerning welfare calculations is in order here. Though the two labour supply systems are indeed identical, the two objective functions are clearly not.

${ }^{13}$ There will be as many macro-agents as there are socio-economic characteristics of interest in the micro-data-base. This could suggest that, without restrictions on the number of these characteristics, we would rapidly run into the "curse of dimensionality" in the general equilibrium set-up, which would of course drastically limit the appeal of the current approach. Fortunately, this is not the case. Indeed, it is possible to adopt identical and standard homothetic intertemporal preferences, and aggregate further these representative labour-supplying agents into a single (per-generation) representative consumer that optimally allocates his/her wealth to lifetime consumption.
} 
subscript to the labour supply of aggregate agents: $L_{i, g, s, t}^{\text {sup }}$.

The OLG structure we use is fairly standard. ${ }^{14}$ We distinguish between $g=1, \ldots, 8$ generations that coexist at each time period $t$ (age groups are: 15-24, 25-34,.., 85-94). The first five age groups are active, while the other three are exogenously retired from the labour force. At the end of each period, the oldest group disappears, a fraction of people belonging to the other age groups die, and a new generation enters the active population according to the following rules:

$$
\begin{aligned}
N_{1, s, t+1} & =\eta_{t} \cdot N_{1, s, t} \\
N_{g+1, s, t+1} & =\Gamma_{g, t} \cdot N_{g, s, t}
\end{aligned}
$$

where $N_{g, s, t}$ denotes the size of the population cell by age and sex at time $t, \eta_{t}$ is an exogenous gross reproduction rate, and $\Gamma_{g, t}$ is the conditional survival probability differentiated by age. Each macro-agent (a) decides how much to work, and in which profession, as was described in section 3.2 above; (b) conditional on this labour force participation decision, he/she chooses the intertemporal profile of consumption (and therefore of asset accumulation) subject to his/her wealth constraint. ${ }^{15}$ Formally, the expected lifetime utility for the generation with gender $s$ that becomes active at time $t$ is assumed of the following form:

$$
U_{s, t}=\sum_{g} \mathfrak{R}^{g-1} \cdot \ln C_{g, s, t+g-1} \cdot \prod_{q=1}^{g} \Gamma_{q, t+q-1}
$$

where $\mathfrak{R}$ is an exogenous discount factor and $C_{g, s, t}$ is consumption. In order to avoid the presence of involuntary bequests, we introduce a life insurance mechanism à la Yaari

\footnotetext{
${ }^{14}$ See e.g. Fougère et al. (forthcoming) for an illustrative use in the context of population ageing. To avoid excessive lengthening of the paper, we only sketch it here. A complete list of equations is available upon request.

${ }^{15}$ Observe that we do not account for intertemporal substitution in leisure over the life-cycle which, from a modern macro perspective, is not entirely satisfactory. This restriction is necessary to preserve the link between the macro and micro frameworks. To avoid such a simplification would require that the microsimulation model be formulated as a dynamic stochastic discrete choice problem, which is extremely more complicated and beyond the scope of this paper. Eckstein and Wolpin (1989a) developed such a life-cycle labour supply model with dynamic stochastic discrete choices, but had also to drastically compromise, by assuming away intertemporal consumption decisions. See Eckstein and Wolpin (1989b) for an introductory survey on dynamic stochastic discrete choice models.
} 
(1965) which implies that the actuarial rate of interest exceeds the market rate by the conditional mortality probability. The intertemporal budget constraint is then:

$$
\begin{aligned}
& \sum_{g} R_{t+g-1} \cdot \prod_{q=1}^{g} \Gamma_{q, t+q-1} \cdot\left[\sum_{i=1}^{I}(1-\varkappa) \cdot A_{i, g, s} \cdot w_{i, t+g-1} \cdot L_{i, g, s, t+g-1}^{\text {sup }}+\text { Pens } s_{g, s, t+g-1}\right] \\
= & \sum_{g} R_{t+g-1} \cdot \prod_{q=1}^{g} \Gamma_{q, t+q-1} \cdot C_{g, s, t+g-1}
\end{aligned}
$$

where $R_{t}$ is the market determined discount factor ${ }^{16}, \varkappa$ is the contributions rate to the pension system, Pens $s_{g, s, t}$ is pension benefit, $w_{i, t}$ is the equilibrium wage (per unit of effective labour) in profession $i$ and $A_{i, g, s}$ is a labour productivity factor that depends on the exogenous characteristics, age $g$ and sex $s$ :

$$
\ln A_{i, g, s}=\varphi_{1, i} \cdot g+\varphi_{2, i} \cdot g^{2}+\varphi_{3, i} \cdot s
$$

Finally, adapting (32), the macro-agent's supply of work-time to profession $i$ is:

$$
\begin{aligned}
L_{i, g, s, t}^{\sup }= & \frac{\left\{\sum_{j} \alpha_{j, g, s}^{-\sigma} \cdot\left[(1-\varkappa) \cdot A_{j, g, s} \cdot w_{j, t}\right]^{\sigma}\right\}^{\frac{\tau}{\sigma}}}{\alpha_{\mathcal{L}, g, s}^{-\tau}+\left\{\sum_{j} \alpha_{j, g, s}^{-\sigma} \cdot\left[(1-\varkappa) \cdot A_{j, g, s} \cdot w_{j, t}\right]^{\sigma}\right\}^{\frac{\tau}{\sigma}}} . \\
& \frac{\alpha_{i, g, s}^{-\sigma} \cdot\left[(1-\varkappa) \cdot A_{i, g, s} \cdot w_{i, t}\right]^{\sigma}}{\sum_{j} \alpha_{j, g, s}^{-\sigma} \cdot\left[(1-\varkappa) \cdot A_{j, g, s} \cdot w_{j, t}\right]^{\sigma}} \cdot N_{g, s, t}
\end{aligned}
$$

The economy produces one good in amount $X$ using physical capital $K$ and effective labour of different profession-types with a constant returns to scale Cobb-Douglas technology:

$$
X_{t}=\prod_{i=1}^{I}\left[L_{i, t}^{d e m}\right]^{\alpha_{i}} \cdot K_{t}^{\beta}
$$

where $L_{i, t}^{d e m}$ is labour demand of each profession by firms. The pension system is Pay-AsYou-Go, with fixed contribution rate $\varkappa$ and endogenous replacement ratio $\gamma_{t}$ determined to balance the pension system budget at each $t$. Pension benefits depend on the average wage earned by the cohort upon retirement:

$$
\begin{gathered}
\text { Pens }_{g, s, t}=\left\{\begin{array}{cc}
\gamma_{t} \cdot \sum_{i=1}^{I} A_{i, g-1, s} \cdot w_{i, t-1} \cdot L_{i, g-1, s, t-1}^{\text {sup }} & g=6 \\
\text { Pens }_{g-1, s, t-1} & g \geqslant 7
\end{array}\right. \\
{ }^{16} R_{t+g-1}= \begin{cases}1 & g=1 \\
\left(\frac{1}{1+r_{t+g-1}}\right) R_{t+g-2} & g \geqslant 2\end{cases}
\end{gathered}
$$


Capital accumulates with net investment assuming constant depreciation rate:

$$
K_{t+1}=I n v_{t}+K_{t} \cdot(1-\delta)
$$

The price system $\left(w_{i, t}, r_{t}\right)$ is determined so that markets balance at each time period:

$$
\begin{aligned}
X_{t} & =\sum_{g} \sum_{s} C_{g, s, t}+\text { Inv }_{t} \\
L_{i, t}^{d e m} & =\sum_{g} \sum_{s} A_{i, g, s} \cdot L_{i, g, s, t}^{\mathrm{sup}}
\end{aligned}
$$

\section{The dynamics of income distribution in an ageing popu- lation: an illustrative example}

In this section, we test the accurateness of the aggregation procedure, and illustrate its usefulness for assessing the dynamics of income distribution. To make a consistent use of both the microsimulation set-up - keep track of individuals - and the general equilibrium model we computer-generate a plausible artificial micro-data set of 51,850 individuals, among which 39,525 aged 15-64 make leisure/work decisions, and choose one of two possible professions (noted Prof-0 and Prof-1). We then link this to an OLG structure calibrated on the fictitious macro data set of an archetype OECD economy. Assuming the dynamic economy is initially stationary, we submit it to a quite drastic demographic shock and compute the equilibrium path of factor rewards. These prices are then plugged into the microsimulation model, and the new optimal discrete choices are computed for each individual, as well as the (by construction: general equilibrium) income levels they earn. We then can assess the dynamics of income distribution and inequalities induced by the demographic change.

\subsection{The micro-data set}

In this initially stationary population, we distinguish individuals by gender and age groups of ten years each, starting at age 15. Only those belonging to the first five cohorts have discrete choices to make: to work or not, and if yes, in which profession. Those from the last three generations are exogenously retired from the labour force. There are 51,850 individuals, each belonging to one specific cell of characteristics, in proportions conveyed 
by Table 1. The declining number of individuals with age reflects transition probabilities $\Gamma_{g, t}$ between cohorts (with initial values reported in the first column of Table 7).

\begin{tabular}{lcc}
\hline & Males & Females \\
\hline \hline $15-24$ & 4000 & 4500 \\
$25-34$ & 4000 & 4500 \\
$35-44$ & 3800 & 4275 \\
$45-54$ & 3600 & 4050 \\
$55-64$ & 3200 & 3600 \\
$65-74$ & 2800 & 3150 \\
$75-84$ & 2000 & 2250 \\
$85-94$ & 1000 & 1125 \\
\hline Total & 24400 & 27450 \\
\hline
\end{tabular}

Table 1: Number of individuals by age and sex in the micro data-set

Within-cell average wages in each profession reflect labour productivity that depends on age and sex; they are generated using an equation consistent with (38):

$$
\ln \bar{w}_{i, g, s}=\varphi_{0 i}+\varphi_{1 i} \cdot g+\varphi_{2 i} \cdot g^{2}+\varphi_{3 i} \cdot s
$$

where $s$ is equal to 0 for males and 1 for females and $g$ equals $1, \ldots, 5$. The parameters adopted for this wage equation are reported in Table 2. The quadratic term is of course meant to capture the hump-shape of labour productivity with respect to age.

\begin{tabular}{lcc}
\hline & Prof-0 & Prof- 1 \\
\hline \hline constant & 5.000 & 5.500 \\
$g$ & 0.400 & 0.450 \\
$g^{2}$ & -0.030 & -0.035 \\
$s$ & -0.300 & -0.320 \\
\hline
\end{tabular}

Table 2: The parameters of the wage equations

Individual wages $w_{i, g, s}^{h}$ are generated, in log, by adding to the log of the average levels $\bar{w}_{i, g, s}$ a normally distributed idiosyncratic productivity term with zero mean and standard deviation $\sigma_{i}=0.5$. General statistics on $w_{i, g, s}^{h}$ are reported in Table 3a and Table $\mathbf{3 b}$. Observe that the standard deviations are chosen quite high so as to make meaningful the accuracy test performed in section 4.3.1. 


\begin{tabular}{lccccc|ccccc}
\hline & \multicolumn{5}{c|}{ Males } & \multicolumn{4}{c}{ Females } \\
\hline \hline $15-24$ & Obs & Mean & Std. Dev. & Min & Max & Obs & Mean & Std. Dev. & Min & Max \\
$25-34$ & 2335 & 222.668 & 114.057 & 37.206 & 994.496 & 2416 & 165.755 & 83.429 & 35.147 & 725.862 \\
$35-44$ & 2393 & 298.008 & 148.925 & 54.594 & 1586.799 & 2582 & 225.667 & 118.394 & 41.730 & 972.842 \\
$45-54$ & 2192 & 386.735 & 196.312 & 47.203 & 1767.226 & 2453 & 287.372 & 143.336 & 51.962 & 1258.786 \\
$55-64$ & 1976 & 475.490 & 240.257 & 69.690 & 2424.641 & 2233 & 346.310 & 175.671 & 55.017 & 1863.712 \\
\hline
\end{tabular}

Table 3a: General statistics on individual wages by age and sex for Prof-0

\begin{tabular}{cccccc|ccccc} 
& \multicolumn{5}{c}{ Males } & \multicolumn{4}{c}{ Females } \\
\hline \hline $15-24$ & Obs & Mean & Std. Dev. & Min & Max & Obs & Mean & Std. Dev. & Min & Max \\
$25-34$ & 872 & 429.900 & 213.679 & 107.592 & 1872.320 & 775 & 325.198 & 157.753 & 80.321 & 1345.630 \\
$35-44$ & 982 & 620.612 & 311.012 & 87.031 & 2378.202 & 969 & 455.526 & 213.806 & 83.345 & 1765.826 \\
$45-54$ & 1158 & 767.865 & 384.479 & 182.279 & 3267.305 & 1017 & 591.654 & 297.682 & 118.489 & 2442.261 \\
$55-64$ & 1217 & 928.794 & 504.224 & 186.391 & 7067.726 & 1057 & 704.562 & 342.523 & 140.431 & 2455.936 \\
\hline
\end{tabular}

Table 3b: General statistics on individual wages by age and sex for Prof-1

Intra-cell individual heterogeneity in preferences is then generated using stochastic terms from generalised extreme-value distribution (9) consistent with a two-level nested multinomial logit with dispersion parameters $\mu_{1}$ and $\mu_{2}$. The inverse of these dispersion parameters are the transformation elasticities $\tau, \sigma$, respectively between leisure and work and between professions (see (33)), the values of which are reported in Table 4 .

\begin{tabular}{lcc}
\hline & Leisure / Work & Prof-0 / Prof-1 \\
\hline \hline Males & & \\
$15-24$ & 0.900 & 1.665 \\
$25-34$ & 0.800 & 1.590 \\
$35-44$ & 0.700 & 1.490 \\
$45-54$ & 0.600 & 1.450 \\
$55-64$ & 0.500 & 1.375 \\
\hline Females & & \\
$15-24$ & 0.850 & 1.700 \\
$25-34$ & 0.750 & 1.625 \\
$35-44$ & 0.650 & 1.575 \\
$45-54$ & 0.550 & 1.540 \\
$55-64$ & 0.450 & 1.475 \\
\hline
\end{tabular}

Table 4: Transformation elasticities of the aggregate labour supply systems

Finally, the preference parameters $\theta_{i}, \theta_{A_{0}}$ of (19) and (21) are chosen so as to generate realistic shares of leisure and work, and arbitrary activity shares by professions: see Table 5. 


\begin{tabular}{lcccc}
\hline & Leisure / Total & Work / Total & Prof-0 / Work & Prof-1 / Work \\
\hline \hline Males & & & & \\
\hline $15-24$ & $19.8 \%$ & $80.2 \%$ & $72.8 \%$ & $27.2 \%$ \\
$25-34$ & $15.6 \%$ & $84.4 \%$ & $70.9 \%$ & $29.1 \%$ \\
$35-44$ & $11.8 \%$ & $88.2 \%$ & $65.4 \%$ & $34.6 \%$ \\
$45-54$ & $11.3 \%$ & $88.7 \%$ & $61.9 \%$ & $38.1 \%$ \\
$55-64$ & $24.0 \%$ & $76.0 \%$ & $56.5 \%$ & $43.5 \%$ \\
\hline Females & & & \\
\hline $15-24$ & $29.1 \%$ & $70.9 \%$ & $75.7 \%$ & $24.3 \%$ \\
$25-34$ & $21.1 \%$ & $78.9 \%$ & $72.7 \%$ & $27.3 \%$ \\
$35-44$ & $18.8 \%$ & $81.2 \%$ & $70.7 \%$ & $29.3 \%$ \\
$45-54$ & $18.8 \%$ & $81.2 \%$ & $67.9 \%$ & $32.1 \%$ \\
$55-64$ & $34.0 \%$ & $66.0 \%$ & $66.7 \%$ & $33.3 \%$ \\
\hline
\end{tabular}

Table 5: Leisure/work rates, and activity rates by profession

\subsection{The macro data set and the ageing shock}

The main parameters and data of the macro model are summarised in Table 6 .

\begin{tabular}{lc}
\hline Consumption / GDP & $80.0 \%$ \\
Investment / GDP & $20.0 \%$ \\
Capital income / GDP & $33.3 \%$ \\
Labour income from Prof-0 / GDP & $35.0 \%$ \\
Labour income from Prof-1 / GDP & $31.7 \%$ \\
Social security contribution rate & $20.0 \%$ \\
Interest rate & $3.3 \%$ \\
Depreciation rate & $5.0 \%$ \\
\hline
\end{tabular}

Table 6: The main parameter values used in the OLG model

The ageing shock is implemented as a temporary fall of the fertility rate $\eta_{t}$ in (34) jointly with the permanent rise in survival rates $\Gamma_{g, t}$ in (35), as reported in Table 7 .

\begin{tabular}{lccccccccccc}
\hline & 1 & 2 & 3 & 4 & 5 & 6 & 7 & 8 & 9 & $>9$ \\
\hline \hline$\eta$ & 1.000 & 1.000 & 1.000 & 0.930 & 0.925 & 0.919 & 0.911 & 0.903 & 0.892 & 1.000 \\
$\Gamma_{15-24}$ & 1.000 & 1.000 & 1.000 & 1.000 & 1.000 & 1.000 & 1.000 & 1.000 & 1.000 & 1.000 \\
$\Gamma_{25-34}$ & 0.950 & 0.950 & 0.950 & 0.950 & 0.950 & 0.950 & 0.950 & 0.950 & 0.950 & 0.950 \\
$\Gamma_{35-44}$ & 0.947 & 0.947 & 0.947 & 0.947 & 0.947 & 0.947 & 0.947 & 0.947 & 0.947 & 0.947 \\
$\Gamma_{45-54}$ & 0.889 & 0.889 & 0.889 & 0.889 & 0.895 & 0.901 & 0.907 & 0.912 & 0.919 & 0.918 \\
$\Gamma_{55-64}$ & 0.875 & 0.875 & 0.875 & 0.875 & 0.882 & 0.890 & 0.897 & 0.904 & 0.912 & 0.911 \\
$\Gamma_{65-74}$ & 0.714 & 0.714 & 0.714 & 0.714 & 0.750 & 0.786 & 0.821 & 0.857 & 0.893 & 0.893 \\
$\Gamma_{75-84}$ & 0.500 & 0.500 & 0.500 & 0.500 & 0.563 & 0.625 & 0.688 & 0.750 & 0.812 & 0.813 \\
\hline
\end{tabular}

Table 7: The demographic shock: time profiles of fertility and survival rates 
The resulting time-path of the population and of the old-age dependency ratio (the ratio of the number of people aged more than 65 to the working age population) are displayed in Figure 1 and Figure 2. ${ }^{17}$ This is indeed a quite drastic ageing shock. The reason for choosing an admittedly excessive demographic change is for testing purposes: we want to generate significant factor-price changes and hence, induce significant switches in individual discrete decisions: only then can we truly gain confidence in the aggregation methodology.

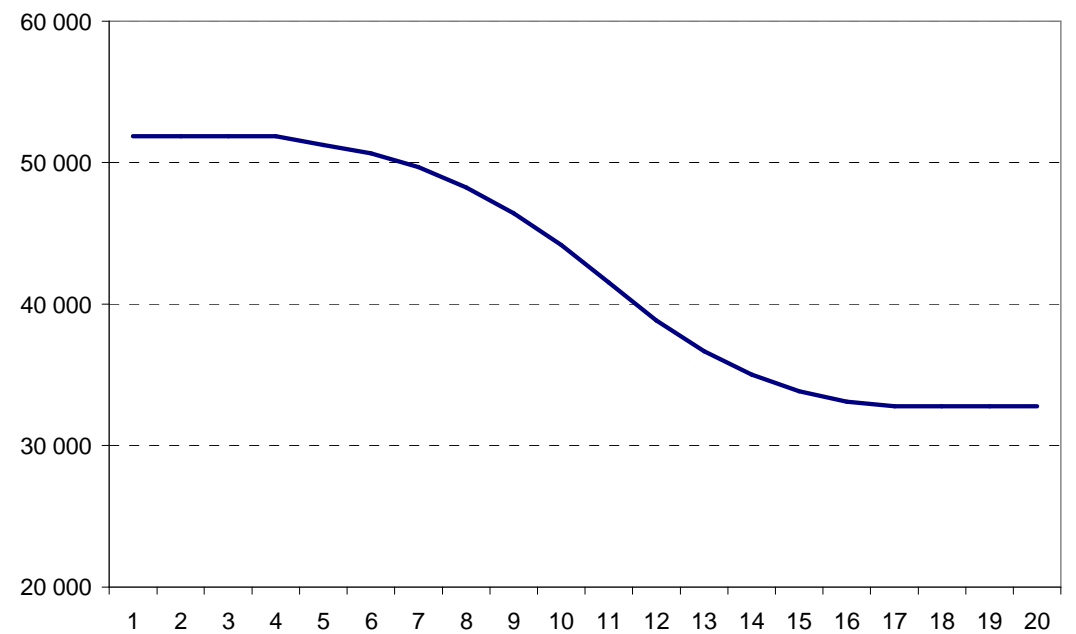

Figure 1: The demographic shock: the time path of total population

\footnotetext{
${ }^{17}$ The long-term change in the old-age dependency ratio of course reflects the permanent rise in the population's life expectancy. Note that the somewhat brutal return of $\eta$ to its initial (unit) level at time-period 10 can be interpreted as resulting from a change in immigration policy.
} 


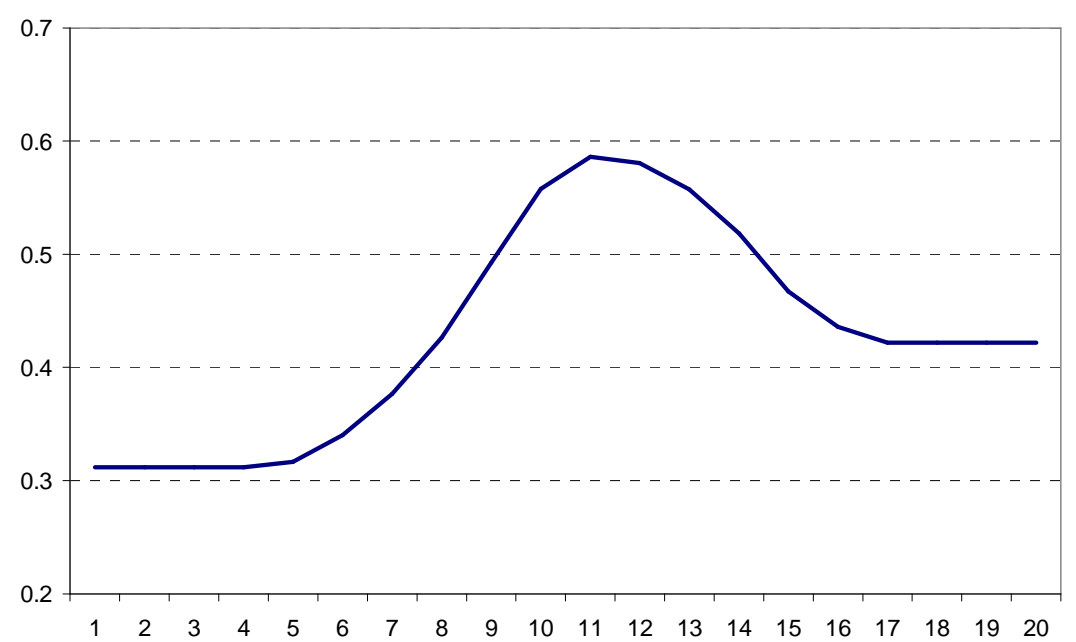

Figure 2: The time path of the old-age dependency ratio

Plugging this changed demographic profile into the OLG model and solving yields, among other things, the time path of equilibrium factor prices which are displayed in Figure 3. ${ }^{18}$ These are as one expects (see e.g. Fougère et al., 2007): the ageing phenomenon results in a temporary rise of the capital-labour ratio that induces equilibrium wage increases in both professions and depresses the equilibrium interest rate. The fact that the equilibrium wage in Prof-0 increases less-and more slowly-than the other, is related to the shift with age of the discrete-choice preferences between professions (see Table 5). Indeed, as they get older, both males and females tend to increasingly value Prof-1, so that the fertility slowdown and the increase in survival probabilities affect more intensely the aggregate labour supply in Prof-0. Figure 4 displays the changes imposed on the equilibrium replacement ratio $\gamma_{t}$ by the demographic shock and the requirement of a balanced budget for the pension system. ${ }^{19}$

\footnotetext{
${ }^{18}$ We only report the first 20 periods though the model is solved over a horizon of 80 periods of ten years each.

${ }^{19}$ The non-smoothness of this time profile of course reflects the fact that the demographic shock is a composite of two effects resulting from the simultaneous change of $\eta$ and $\Gamma$.
} 


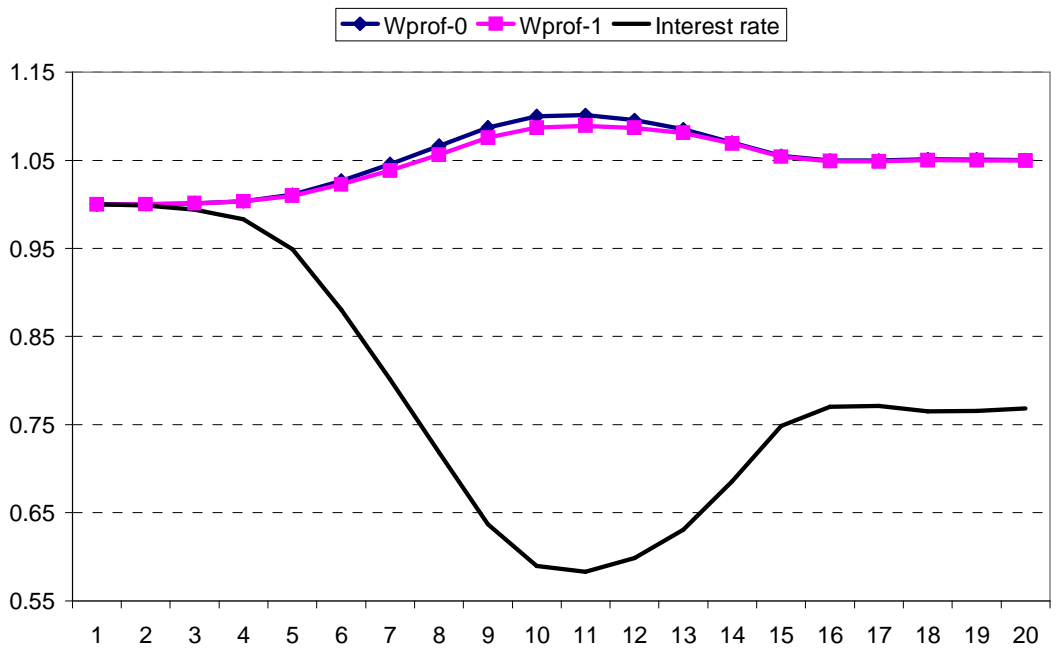

Figure 3: The dynamics of factor prices induced by the demographic shock

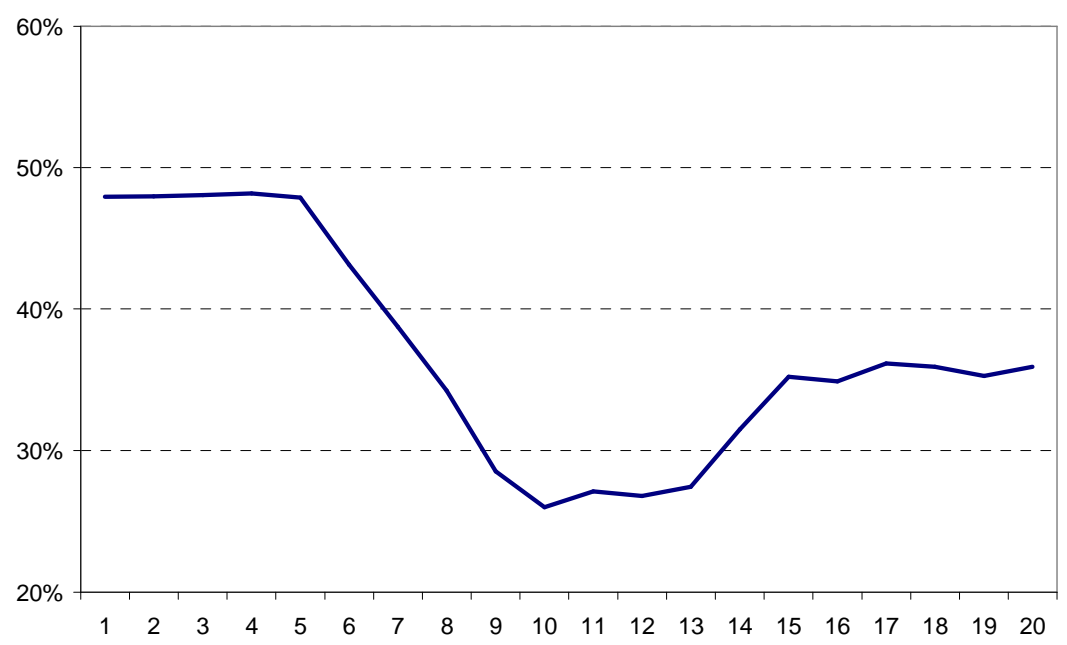

Figure 4: The dynamics of the replacement ratio induced by the demographic shock

\subsection{Microsimulation results}

Now that the general equilibrium time-path of factor prices consistent with the new demographics has been computed, we plug these prices into the behavioural microsimulation model. We determine the optimal discrete choices of each of the micro-agents aged 15-64 when they face the new economic environment, and compute the resulting earned income for each of them. We first check the accuracy of the aggregation methodology, and then report income distribution statistics. 


\subsubsection{Accuracy}

We check the accuracy of the aggregation procedure by computing the individual discrete labour-supply decision for each of the 39,525 individuals aged 15-64 facing the new general equilibrium factor prices. We then sum, within each population cell, the labour supplies and compare with those generated from the representative agent formulation in the OLG model (that is, those used to generate the equilibrium factor price paths). Why could these predictions differ, given that we use exact aggregation results? It will be remembered that, within each population cell, we assumed that labour supply decisions are made using-both in the micro and in the macro approach the within-cell average wage rather than the true ex-post individual wages $w_{i, g, s}^{h}$, the latter being adjusted for within-cell idiosyncratic productivity differences. The microsimulation computations use this information on individual productivity differences. Making sure that the resulting discrepancies are small is therefore indeed meaningful.

We find that the largest percentage discrepancy between the macro- and micro- predicted labour supplies is lower than $0.01 \%$ per cent, a very small number given the severity of the demographic shock: clearly, a discrepancy that is unlikely to affect the equilibrium wages and is therefore without general equilibrium significance.

\subsubsection{Income distribution effects of population ageing}

Having checked the quality of the aggregation procedure, we are now set to report on how the ongoing ageing of our economies may affect income inequalities, thanks to the microsimulations model. Various inequality indices are available in the literature, each with its pros and cons; given that our data are computer generated and the exercise more illustrative than applied to a real world case, we limit ourselves to two of these without apologies.

In Figure 5, we report the median, tenth percentile, and ninetieth percentile of the income distribution for the entire population. ${ }^{20}$

The negative dynamics of the median and ninetieth percentiles are easy to understand

\footnotetext{
${ }^{20}$ The individual incomes are therefore computed as the sum of three components: the labour income (net of social security contributions), the capital income, and the pension benefits.
} 
from the time path of factor prices and of the replacement ratio, respectively reported in Figures 3 and 4 . The former individual is either a middle-aged low-skilled individual -i.e. an individual working in the low-wage profession Prof-0 who benefits from increasing wages but simultaneously suffers from decreasing rents on his accumulated assets- or a middle income retired individual whose earnings suffer from both decreasing pension benefits and depressed capital rental rates. The ninetieth percentile individual is either a middle-aged qualified worker, or a high income retired individual, both with significant shares of their income due to accumulated capital assets.

The evolution of the tenth percentile of income directly reflects the dynamics of wages: this individual is either a young low-skilled individual who benefits from higher wages in Prof-0, or a previously unemployed whose reservation wage falls short of the new equilibrium rate and who therefore chooses to enter the labour market; in both cases this tenth-percentile individual is only mildly affected by the (permanently) depressed interest rates.

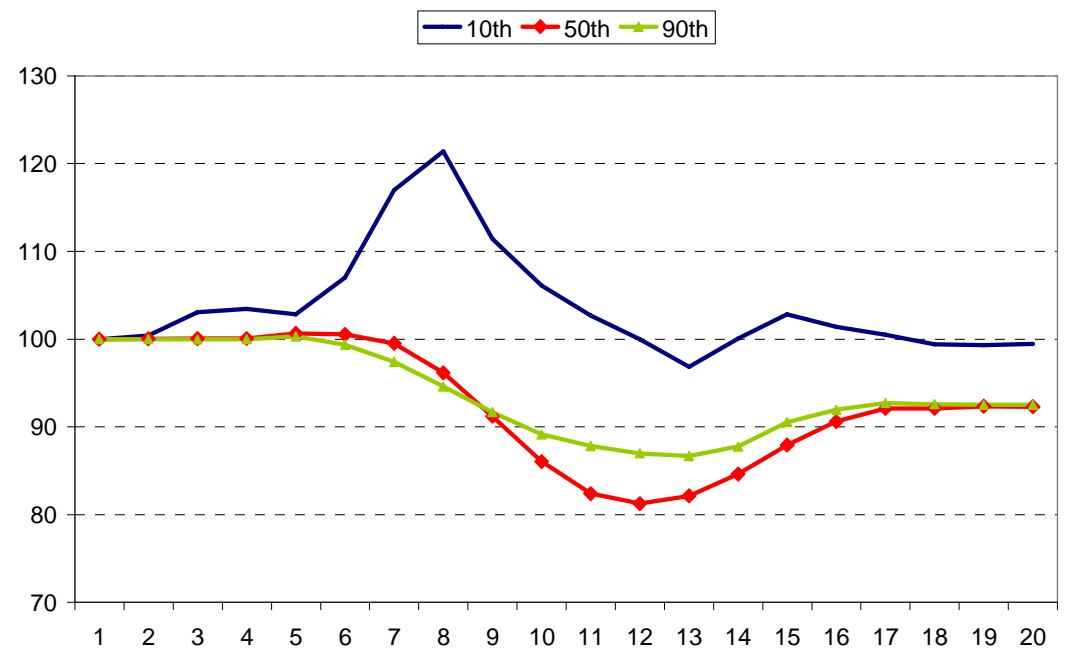

Figure 5: The time path of the $10^{t h}, 50^{t h}$, and $90^{t h}$ percentiles

In Figure 6, $\mathbf{7}$ and 8, are displayed the time path of the Gini coefficients for age-groups 15-24, 55-64 and 65-94, which are the most contrasted.

The first age group is quite specific in that it holds no previously accumulated asset so that its flow income is independent of interest rate fluctuations. Income inequality 
unambiguously decreases for this group thanks to rising wages and the resulting boost of the participation rate of both men and women, as some individuals previously inactive decide to step into the job market. Consistently, given that equilibrium wages remain permanently above their initial level, income inequality reduction remains true in the long run for young adults.

As one expects, things are slightly more complicated when one considers the 55-64 cohort because returns on accumulated assets are here likely to represent a larger share of income for some. Individuals who have accumulated large financial portfolios in the past experience a significant capital income drop, while those who own little wealth but place a high value on work will benefit unambiguously from the factor price changes.

The time path of the Gini index for people aged more than 65 is much more complex to decrypt because it results from three possibly conflicting forces. First, returns to capital fall, and this will hurt some much more than others; second, the equilibrium replacement ratio is drastically reduced (see Figure (4)) which, for given wages, implies a significant downscaling of pension revenues; third, this contribution rate applies to different wages depending on the age cohort (see equation (39)) so that some can benefit while others might on the contrary suffer from the equilibrium wage fluctuations.

Observe that this assessment of the way individuals, depending on their socio-economic characteristics, will share the costs and benefits of the change in the demographic trend strongly depends on our assumption that the pension system is pay-as-you-go with budget balanced at every period by an endogenous replacement ratio $\gamma_{t}$. Other policy scenarios -such as letting the contribution rate $\varkappa$ adjust rather than the replacement ratio, or smoothing the transitional effects through bond financing- as well as other social institutions -such as switching from pay-as-you-go to full capitalisation- could of course be explored, and their impact on income inequalities quantified. Such an exploration of the dynamics of income inequalities following an ageing shock would not be meaningful without making use of two consistent microsimulation and a general equilibrium models: the methodology developed in this paper ensures that this consistency is feasible and easy to implement. 


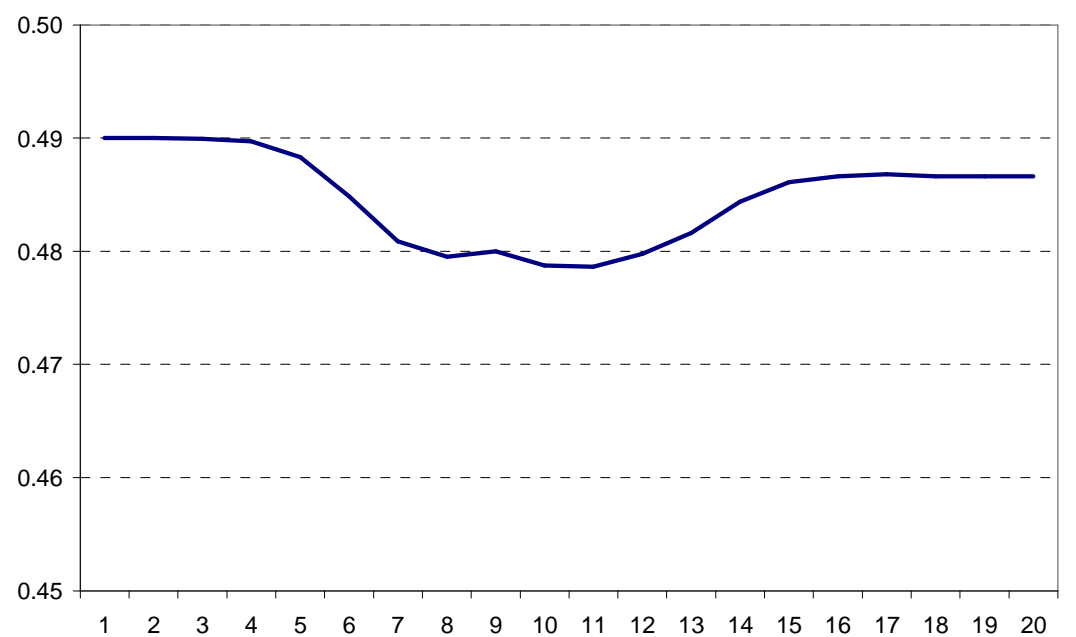

Figure 6: The time path of the Gini index for the age group 15-24

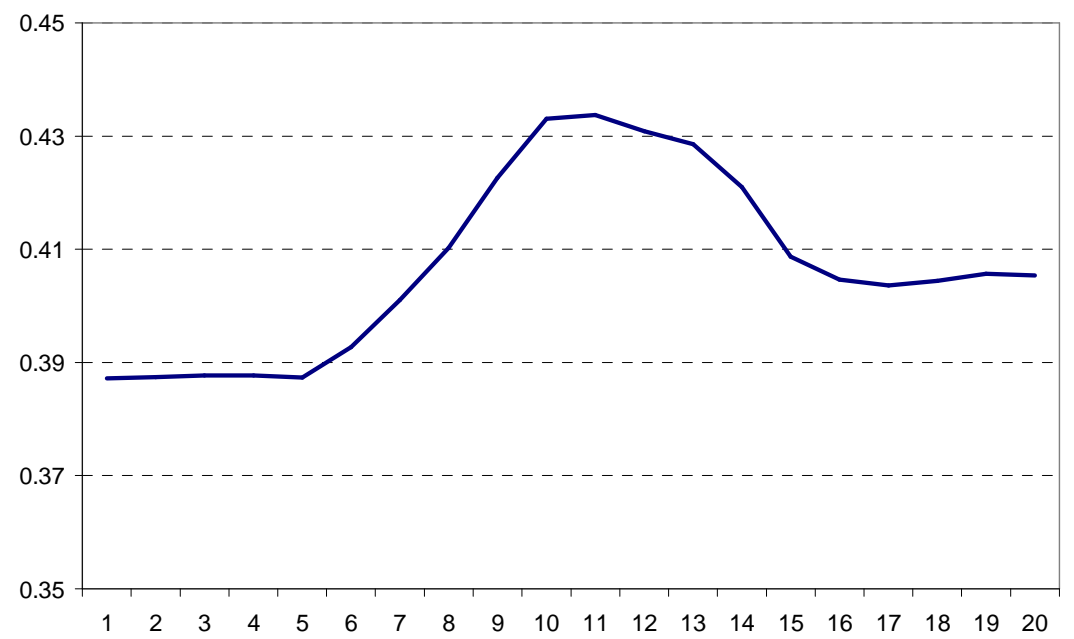

Figure 7: The time path of the Gini index for the age group 55-64 


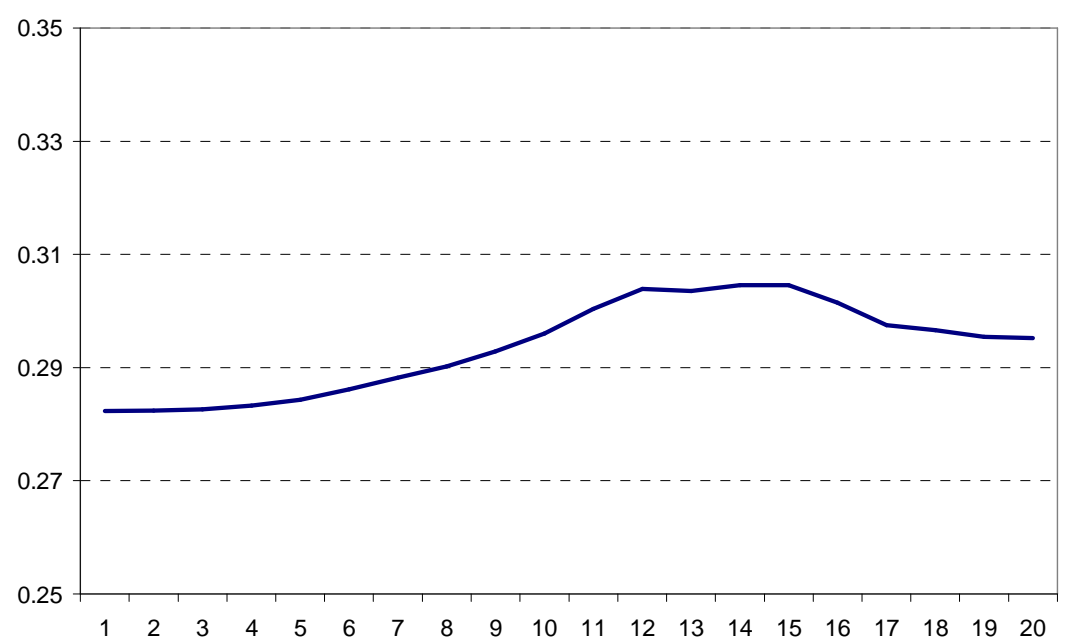

Figure 8: The time path of the Gini index for the age group 65-94

\section{Conclusion}

Computable general equilibrium models have become indispensable tools of quantitative policy assessment. By essence, they rely on some form of representative agents simplification of the economy necessary to make explicit and manageable the consistency imposed on individual decisions by technological and resource constraints. As such, they are unable to keep track of individual heterogeneities that affect decisions at the underlying micro level. As huge micro-data sets have increasingly been made available in recent years, the microsimulation approach has gained popularity precisely because it takes into account the full heterogeneity of individual adjustments to policy reforms. In these models, individual decision-making is often made over a set of discrete alternatives. But this is typically a partial equilibrium approach that sacrifices global consistency. Iterations between the two frameworks is always possible, but bound to be at best tedious, possibly inaccurate or unreliable if the convergence path is ill behaved.

We have suggested in this paper a bridge between the two model types by making use of some exact aggregation results that provide an interface between the two approaches. Many reasons can be mentioned that advocate for the usefulness of these aggregation results. First, it is likely that working with a well behaved aggregate agent is conceptually much easier and convenient to many modellers, when analysing GE policy results, than 
thinking in terms of random utility discrete choices of myriads of micro-agents. Second, the theoretical characterisation of the properties of a general equilibrium (such as existence and uniqueness of a solution, or the convergence properties of a fixed-point algorithm) is likely to be much easier if one can rely on well behaved preferences of macro-agents rather than deal with myriads of heterogeneous dichotomous choice-making micro-agents. Third, by avoiding the drawbacks mentioned above of an iterative procedure between the two frameworks, they make computations more accurate.

The aggregation results can also prove useful to CGE modellers not interested in the articulation between their and the behavioural microsimulation approach. Indeed, the explosion of the microeconometric literature during the last two decades provides us with empirical estimates drawn from huge data sets of individual data, a large fraction of which uses some form of the nested logit model. Making use of this econometric information on preferences and/or technologies can only improve the quality of the GE predictions. We have shown how CGE modellers can easily take advantage of such empirical information with little methodological cost.

Potential applications of the aggregation methodology introduced in this paper are numerous. Income inequality issues is one, as was illustrated in a dynamic setting, by linking a microsimulation model built from a computer-generated data set to a calibrated OLG general equilibrium representation of an economy submitted to demographic ageing.

\section{References}

[1] Anderson S., A. de Palma and J-F. Thisse (1992), Discrete Choice Theory of Product Differentiation. Cambridge: MIT Press.

[2] Arntz M., S. Boeters and N. Gurtzgen (2006), "Alternative Approaches to Discrete Working Time Choice in an AGE Framework". Economic Modelling, 23(6), 1008-1032.

[3] Arntz M., S. Boeters, N. Gurtzgen and S. Schubert (2008), "Analysing Welfare Reform in a Microsimulation-AGE Model: The Value of Disaggregation". Economic Modelling, 25(3), $422-439$. 
[4] Ben-Akiva M. and S. Lerman (1985), Discrete Choice Analysis: Theory and Application to Predict Travel Demand. Cambridge: MIT Press.

[5] Bourguignon F. and A. Spadaro (2006), "Microsimulation as a Tool for Evaluating Redistribution Policies". Journal of Economic Inequality, 4(1), 77-106.

[6] Eckstein Z. and K. Wolpin (1989a),"Dynamic Labour Force Participation of Married Women and Endogenous Work Experience". Review of Economic Studies, 56, 375-390.

[7] Eckstein Z. and K. Wolpin (1989b),"The Specification and Estimation of Dynamic Stochastic Discrete Choice Models: A Survey". Journal of Human Resources, XXIV(4), 562-598.

[8] Fougère M., J. Mercenier and M. Mérette (2007), "A Sectoral and Occupational Analysis of Population ageing in Canada Using a Dynamic CGE Overlapping Generations Model", Economic Modelling 24(4), 690-711.

[9] McFadden D. (1978), "Modeling the Choice of Residential Location". In A. Karlqvist, L. Lundqvist, F. Snickars and J. Weibull (eds), Spatial Interaction Theory and Planning Models. Amsterdam: North-Holland.

[10] McFadden D. (1981), "Econometric Models of Probabilistic Choice". In C. Manski and D. McFadden (eds), Structural Analysis of Discrete Data with Econometric Applications. Cambridge: MIT Press.

[11] Rausch S. and T. Rutherford (2007), "Computation of Equilibria in OLG Models with Many Heterogeneous Households", mimeo.

[12] Savard L. (2003), "Poverty and Income Distribution in a CGE-Household Microsimulation Model: Top-Down/Bottom-Up Approach", CIRPEE WP 03-43.

[13] Train K. (1986), Qualitative Choice Analysis. Cambridge: MIT Press.

[14] Train K. (2003), Discrete Choice Method with Simulation. Cambridge: Cambridge University Press.

[15] Yaari M. (1965), "Uncertain lifetime, life insurance, and the theory of the consumer". Review of Economic Studies, 32, 137-150. 